\title{
Prediction on Affected Upper Extremity Function in Hemiplegic Patients after Thalamic Hemorrhage Using Somatosensory Evoked Magnetic Fields
}

\author{
Hideki YosHIDA $^{1}$, Takeo KONDO ${ }^{2}$ and Nobukazu NAKASATO ${ }^{3}$ \\ ${ }^{1}$ Department of Physical Therapy, School of Health Sciences, Hirosaki University, Aomori 036-8564, Japan \\ ${ }^{2}$ Section of Physical Medicine and Rehabilitation, Department of Disability Science, Tohoku University Graduate School of \\ Medicine, Miyagi 980-8575, Japan \\ ${ }^{3}$ Department of Neurosurgery, Kohnan Hospital, Miyagi 982-8523, Japan
}

\begin{abstract}
The aim of the present study was to investigate the prognostic value of somatosensory evoked magnetic fields (SEFs) at an acute stage on recovery of an affected upper extremity (UE) function as practicality in hemiplegic patients after thalamic hemorrhage. Nine hemiplegic patients after thalamic hemorrhage were enrolled in this study. Median nerve SEFs, evoked by electrical stimulation at the wrist of the affected UE, were measured using a 204 channel whole-head magnetoencephalography system within 72 hours after the onset of thalamic hemorrhage (acute stage). Assessments on the affected UE, which included the motor palsies of the UE and fingers (Brunnstrom's motor recovery stage: BS), sensory disturbance (the thumb localizing test) and UE function (the UE ability test), were performed at both the acute stage and 3 months after the onset of thalamic hemorrhage (chronic stage). Almost all the patients showing any median nerve SEF components that originated from the somatosensory cortex in the affected hemisphere and occurred between about $20 \mathrm{~ms}$ and 100 ms post-stimulus at the acute stage demonstrated good outcomes in the motor palsies (BSV), sensory disturbance (normal) and affected UE function (practical hand) at the chronic stage. In contrast, majority of patients not showing them at all demonstrated poor outcomes in the motor palsies (BSIII or less), sensory disturbance (severely impaired) and affected UE function (disabled hand) at the chronic stage. These results suggest that the findings of the median nerve SEFs at the acute stage would contribute to the early outcome prediction on the affected UE function in hemiplegic patients after thalamic hemorrhage.
\end{abstract}

Key words: somatosensory evoked magnetic fields, thalamic hemorrhage, prediction, affected upper extremity function

(J Jpn Phys Ther Assoc 9: 9-15, 2006)

$\mathbf{T}_{\mathrm{H}}$

halamic hemorrhage accounts for approximately $30 \%$ of spontaneous intracerebral hemorrhage ${ }^{1)}$. Since the thalamus, which is a transmission point of somatosensory information, is located near the posterior limb of the internal capsule including the corticospinal tract, patients after thalamic hemorrhage often demonstrate hemisensory disturbances and/or hemiplegia contralateral to the affected thalamus. As the result, they suffer from various limitations

Received: April 5, 2005

Accepted: November 12, 2005

Correspondence to: Hideki Yoshida, Department of Physical Therapy, School of Health Sciences, Hirosaki University, 66-1 Honcho, Hirosaki-shi, Aomori 036-8564, Japan. in activities of daily living (ADL). Especially, the limitations of ADL (e.g. feeding and grooming, etc.) in which an affected upper extremity (UE) function as practicality plays an important role remarkably reduce patient's quality of life. Therefore, predicting the outcome of the affected UE function at an acute stage is indispensable to rational planning of rehabilitation treatment in hemiplegic patients after thalamic hemorrhage. Nevertheless, most previous studies predicting functional outcomes of hemiplegic patients after thalamic hemorrhage have focused on overall $\mathrm{ADL}^{2-4)}$ and gait ability ${ }^{5)}$, and few studies have addressed the affected UE function ${ }^{6}$. 
Magnetoencephalography (MEG) is widely applied to recent stroke studies. MEG, which measures magnetic fields generated by electrical activities of cerebral cortical neurons, provides a non-invasive evaluation method on brain functions. Since MEG performs source estimation of evoked responses related to sensorimotor, auditory and visual functions with high spatial resolution ${ }^{7) 8}$ ), which is a generally difficult technique in electroencephalographic studies, it has many advantages over electroencephalography in evaluation of brain functions. We previously reported that findings of somatosensory evoked magnetic fields (SEFs) at an acute stage were associated with motor recovery as well as sensory recovery at one month after the onset of thalamic hemorrhage ${ }^{9}$. However, the prognostic value of SEFs for the outcome of the affected UE function in hemiplegic patients after thalamic hemorrhage remained unclear.

The aim of the present study was to investigate the prognostic value of SEFs at an acute stage on recovery of the affected UE function in hemiplegic patients after thalamic hemorrhage.

\section{Subjects and Methods}

\section{Subjects}

Nine hemiplegic patients after thalamic hemorrhage ( 5 females and 4 males, mean 61.4 years old) were enrolled in this study. Patients were included if they were not operated on for thalamic hemorrhage, could understand simple orders and did not have any higher cortical dysfunctions. Demographic and clinical profiles of the patients are presented in Table 1. Written informed consent was obtained from all the patients.

\section{SEF measurement and analysis}

SEFs were measured using a 204 channel whole-head MEG system (Neuromag, Helsinki, Finland) in a magnetically shield room within 72 hours after the onset of thalamic hemorrhage (acute stage). SEF measurement was performed with the patient awake and reclining on a bed with the head inserted into the MEG helmet. In this study, SEFs in the affected hemisphere were measured for current stimulation on the median nerve at the wrist of the affected UE. Stimulus current was monophasic square-wave impulses with $0.3 \mathrm{~ms}$ duration at $2.8 \mathrm{~Hz}$. Stimulus intensity was just above the motor threshold of the abductor pollicis brevis muscle. Conditions for the SEFs data acquisition were sampling at $1001.6 \mathrm{~Hz}$ and band-pass filtering between 0.1 to $260.4 \mathrm{~Hz}$, respectively. The SEF data were averaged 200 times for further analysis.

The median nerve SEF components occurring between about $20 \mathrm{~ms}$ and $100 \mathrm{~ms}$ post-stimulus in the affected hemisphere, which generally include four or five components in healthy subjects ${ }^{10)}$, were chosen for analysis. The median nerve SEF component with the latency of about $20 \mathrm{~ms}$ is the initial one called $\mathrm{N} 20 \mathrm{~m}^{7}$, but systematic classification of subsequent components after $\mathrm{N} 20 \mathrm{~m}$ is not necessarily easy because of their great interindividual variability ${ }^{11) 12}$. In each patient, after peaks of the median nerve SEF components in the above-mentioned latency window were visually identified as the points of maximum amplitude, the source location of only the SEF component with the shortest latency was estimated using a single equivalent current dipole model ${ }^{13)}$. The estimated SEF source was superimposed on the patient's head magnetic resonance (MR) image when the goodness-of-fit ${ }^{14)}$ of the model exceeded $80 \%$, and only the SEF component that originated from the somatosensory cortex in the affected hemisphere was accepted as a reliable response. A MEG-

Table 1. Demographic and clinical profiles of nine hemiplegic patients after thalamic hemorrhage

\begin{tabular}{|c|c|c|c|c|c|c|c|c|c|c|}
\hline \multirow{2}{*}{$\begin{array}{c}\text { Patient } \\
\text { No. } \\
1\end{array}$} & \multirow{2}{*}{$\frac{\text { Age (yr.) }}{65}$} & \multirow{2}{*}{$\frac{\operatorname{Sex}}{\mathrm{M}}$} & \multirow{2}{*}{$\begin{array}{c}\begin{array}{c}\text { Lesion } \\
\text { side }\end{array} \\
\mathrm{R}\end{array}$} & \multirow{2}{*}{$\frac{\mathrm{Vol}(\mathrm{ml})^{¥}}{10.3}$} & \multirow{2}{*}{$\begin{array}{l}\text { Destruction of } \\
\text { the posterior limb of } \\
\text { the internal capsule } \\
-\end{array}$} & \multicolumn{5}{|c|}{$\begin{array}{l}\text { Peak latencies of the median nerve } \\
\text { SEF components (ms) }\end{array}$} \\
\hline & & & & & & NR & & & & \\
\hline 2 & 65 & $\mathrm{~F}$ & $\mathrm{R}$ & 17.3 & + & NR & & & & \\
\hline 3 & 76 & $\mathrm{~F}$ & $\mathrm{~L}$ & 10.6 & + & NR & & & & \\
\hline 4 & 53 & $\mathrm{~F}$ & $\mathrm{~L}$ & 15.4 & - & $23.7^{\$}$ & 68.9 & & & \\
\hline 5 & 49 & M & $\mathrm{L}$ & 16.5 & + & 28.9 & 38.3 & 55.3 & & \\
\hline 6 & 52 & $\mathrm{~F}$ & $\mathrm{R}$ & 7.1 & - & 73.6 & & & & \\
\hline 7 & 70 & $\mathrm{~F}$ & $\mathrm{R}$ & 13.2 & - & $22.7^{\$}$ & 30.3 & 63.7 & & \\
\hline 8 & 52 & $\mathrm{M}$ & $\mathrm{L}$ & 16.1 & + & $21.8^{\$}$ & 29.8 & 48.2 & & \\
\hline 9 & 71 & $\mathrm{M}$ & $\mathrm{L}$ & 5.3 & - & $23.7^{\$}$ & 30.8 & 43.9 & 57.1 & 87.3 \\
\hline
\end{tabular}

M, male; F, female; R, right; L, left; Vol, hematoma volume; -, not destructed; +, destructed. ${ }^{¥}$, the findings indicated by head computed tomographic images taken immediately after the onset of thalamic hemorrhage. NR, no response of $\mathrm{SEFs} ;{ }^{\$}$, the SEF component confirmed as N20m. 
MR imaging cartesian coordinate was integrated on a workstation, using fiduciary points, i.e. the nasion and bilateral preauricular points, on the patient's head.

The SEF measurement and analysis were performed by one physical therapist (H.Y.) under the supervision of neurosurgeons.

\section{Assessments on the affected UE}

Assessments on the affected UE were performed at both the acute stage and a chronic stage. In this study, the chronic stage was defined as 3 months after the onset of thalamic hemorrhage because, in general, the recovery of the affected UE in stroke patients will reach plateau within 3 months after stroke onset, in the aspects of both impairments ${ }^{15)}$ and disabilities ${ }^{16)}$.

The severities of the motor palsies of the affected UE and fingers were assessed using Brunnstrom's motor recovery stage $(\mathrm{BS})^{17)}$, which classified the severities into sequential six recovery stages from I (paralysis) to VI (almost normal). The severity of a sensory disturbance of the affected UE was assessed using the thumb localizing test ${ }^{18)}$, which is a quantitative assessment method for the joint position sensation of the affected UE. In this study, the result of the thumb localizing test was classified into four grades as follows: "normal", a patient can pinch the affected thumb with the non-affected thumb and index finger correctly and quickly; "slightly impaired", a patient can pinch the affected thumb with the non-affected thumb and index finger after the several centimeter fluctuation of the non-affected UE; "severely impaired", a patient can manage to pinch the affected thumb with the non-affected thumb and index finger although the fluctuation of the nonaffected UE is remarkable; "sensory loss", a patient can not pinch the affected thumb with the non-affected thumb and index finger at all. The severity of the affected UE function was assessed using the UE ability test (UEAT) ${ }^{19)}$. UEAT, which contains following five subtests: (1) putting up an umbrella; (2) picking out a coin from a wallet; (3) cutting self-fingernails; (4) holding an envelope when cutting it with scissors; (5) buttoning the cuff of the non-affected side, is a quantitative assessment method for the affected UE function, and makes it possible to classify the severity of the affected UE function into six levels based on the result of the five subtests as follows: "practical hand A", a patient can perform all the subtests; "practical hand B", a patient can perform 4 out of 5 subtests; "assisted hand A", a patient can perform 3 out of 5 subtests; "assisted hand B", a patient can perform 2 out of 5 subtests; "assisted hand C", a patient can perform 1 out of 5 subtest; "disabled hand", a patient can not perform any subtests.

\section{Data analysis}

The patients were classified into two groups, i.e. "response group (R group)" and "no-response group (NR group)", according to findings of the median nerve SEFs: the former consisted of patients showing any median nerve SEF components that originated from the somatosensory cortex in the affected hemisphere and occurred between about $20 \mathrm{~ms}$ and $100 \mathrm{~ms}$ post-stimulus, regardless of the absence of $\mathrm{N} 20 \mathrm{~m}$, and the latter consisted of those not showing them at all. The severities of the motor palsies, sensory disturbance and affected UE function were compared between the two groups at both the acute (baseline) and chronic stages.

Statistical analysis was performed using SPSS 12.0 for Windows, and Mann-Whitney's U test was adopted to compare each assessment item between the two groups. Statistical significance was set at $\mathrm{p}<0.05$.

\section{Results}

\section{SEF analysis}

Table presents peak latencies of the median nerve SEF components in all the patients. As a whole, four patients showed N20m, 2 showed only SEF components after N20m (i.e. the number of the patients in the R group was 6) and 3 did not show any SEF components (i.e. the number of the patients in the NR group was 3). The median nerve SEFs were observed even in some of the patients in whom destruction of the posterior limb of the internal capsule including the somatosensory tract was indicated by head computed tomographic images taken immediately after the onset of thalamic hemorrhage (Patient 5 and 8: see Table 1). Figure 1 presents the median nerve SEF representative waveforms, components occurring between about $20 \mathrm{~ms}$ and $100 \mathrm{~ms}$ post-stimulus and source locations of the components with the shortest latency in the patients of the $\mathrm{R}$ group. In all of them, the SEF component with the shortest latency was accepted as a reliable response because the estimated source was located on the posterior bank of the central sulcus, i.e. the somatosensory cortex, in the affected hemisphere.

\section{SEFs and the motor palsies of the affected UE and fingers}

Figures 2 and Fig. 3 present the relationship between the findings of the median nerve SEFs at the acute stage and the severities of the motor palsies of the affected UE and fingers at both the acute and chronic stages. In the R group, although the motor palsies of both the affected UE and fingers showed various severities at the acute stage, they recovered to BSV in all the patients without one (Patient 7) at the chronic stage. In the NR group, on the other hand, the motor palsies of both the affected UE and fingers remained BSIII or less in all the patients without one (Patient 3) from the acute to chronic stages. In the statistical analysis, the severity of the motor palsy of the affected UE in the R group was significantly lower than that in the NR group at the acute stage. However, the severities of the motor 

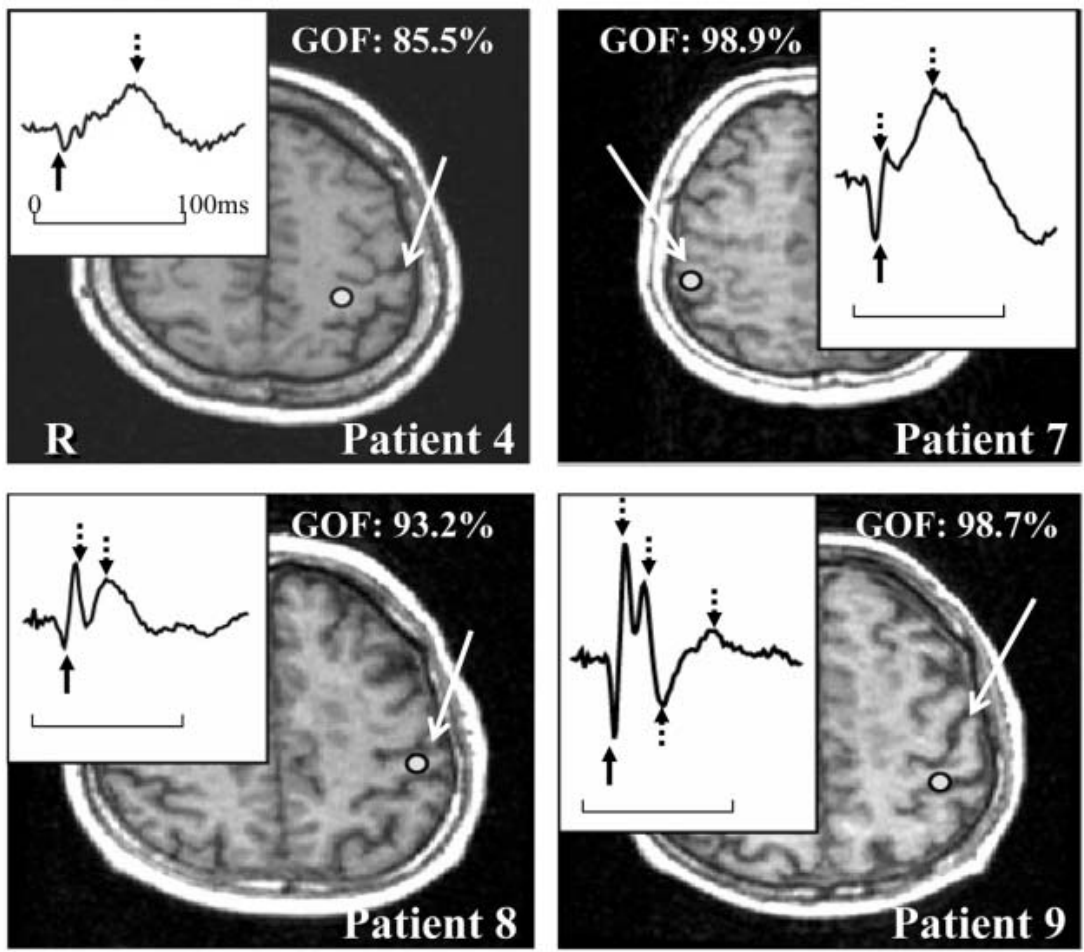

N20m (+)
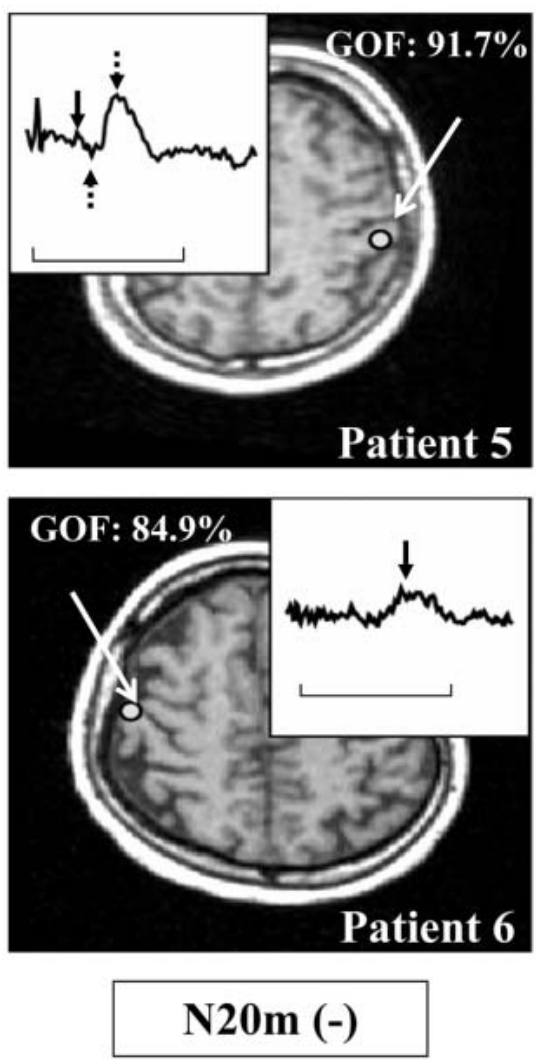

Fig. 1. The median nerve SEF representative waveforms, components occurring between about $20 \mathrm{~ms}$ and $100 \mathrm{~ms}$ post-stimulus and source locations of the components with the shortest latency in the patients of the $\mathrm{R}$ group. Black arrows point to the median nerve SEF components with the shortest latency. Black arrows with a dashed line point to the median nerve SEF components after ones with the shortest latency. Circles represent the source locations of the median nerve SEF components with the shortest latency. White arrows point to the central sulcus. GOF, goodness-of-fit.

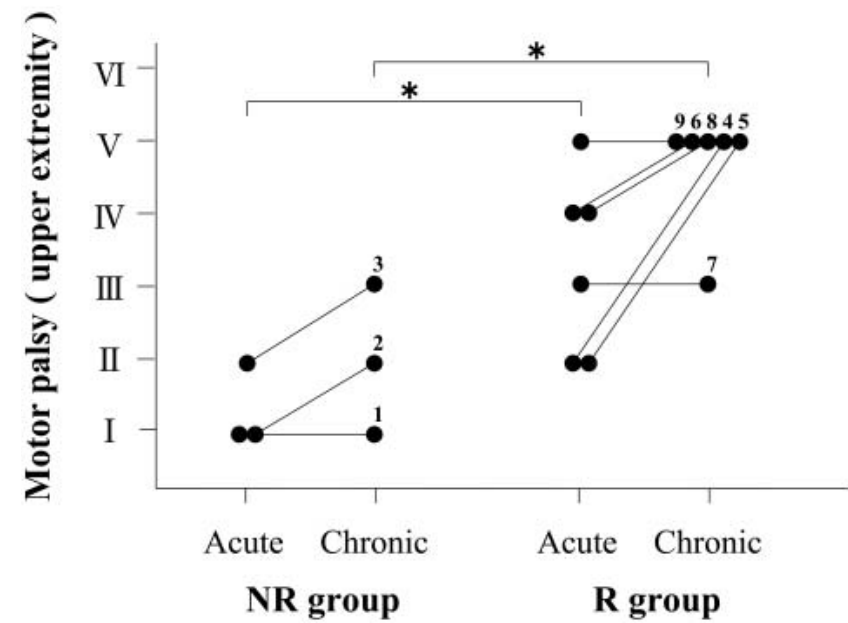

Fig. 2. Relationship between the findings of the median nerve SEFs at the acute stage and the severity of the motor palsy of the affected upper extremity at both the acute and chronic stages. Each number in Fig. 2 represents Patient No. ${ }^{*}, \mathrm{p}<0.05$.

palsies of both the affected UE and fingers in the R group were significantly lower than those in the NR group at the chronic stage.

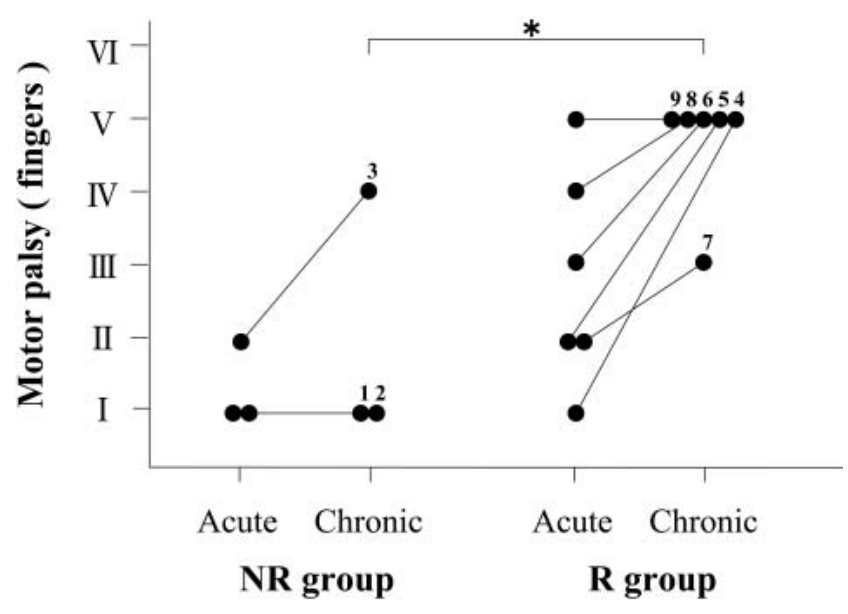

Fig. 3. Relationship between the findings of the median nerve SEFs at the acute stage and the severity of the motor palsy of the affected fingers at both the acute and chronic stages. Each number in Fig. 3 represents Patient No. $*, \mathrm{p}<0.05$.

SEFs and the sensory disturbance of the affected UE

Figure 4 presents the relationship between the findings of the median nerve SEFs at the acute stage and the severity of the sensory disturbance of the affected UE at both the 


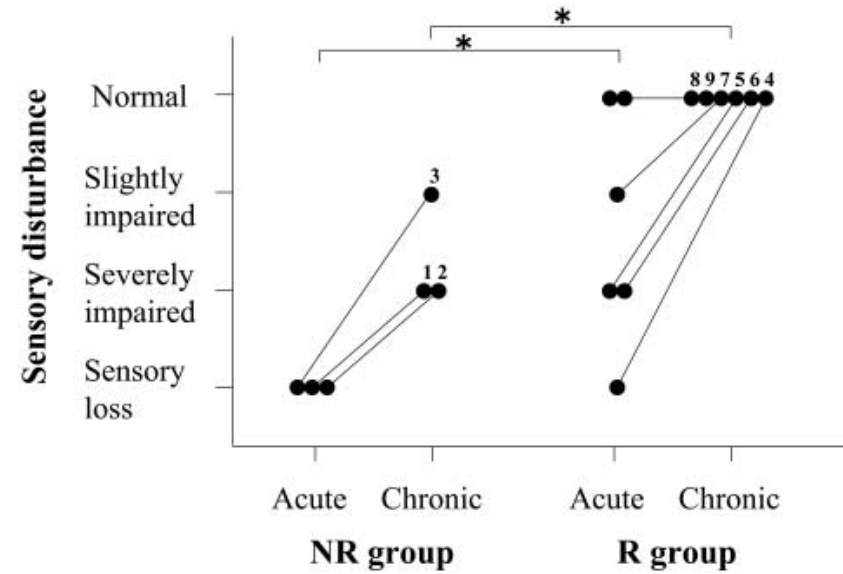

Fig. 4. Relationship between the findings of the median nerve SEFs at the acute stage and the severity of the sensory disturbance of the affected upper extremity at both the acute and chronic stages. Each number in Fig. 4 represents Patient No. ${ }^{*}, \mathrm{p}<0.05$.

acute and chronic stages. In the $\mathrm{R}$ group, although the sensory disturbance of the affected UE showed various severities at the acute stage, it recovered to "normal" in all the patients at the chronic stage. In the NR group, on the other hand, the sensory disturbance of the affected UE showed "sensory loss" in all the patients at the acute stage, and it recovered only to "severely impaired" or "slightly impaired" at the chronic stage. In the statistical analysis, the severity of the sensory disturbance of the affected UE in the $\mathrm{R}$ group was significantly lower than that in the NR group at both the acute and chronic stages.

\section{SEFs and the affected UE function}

Figure 5 presents the relationship between the findings of the median nerve SEFs at the acute stage and the severity of the affected UE function at both the acute and chronic stages. In the R group, although the affected UE function showed various severities at the acute stage, it recovered to "practical hand A" in all the patients without one (Patient 7) at the chronic stage. In the NR group, on the other hand, the affected UE function showed "disabled hand" in all the patients at the acute stage, and it remained "disabled hand" or recovered only to "assisted hand B" at the chronic stage. In the statistical analysis, no significant difference on the severity of the affected UE function was observed between the two groups at the acute stage. However, the severity of the affected UE function in the R group was significantly lower than that in the NR group at the chronic stage.

\section{Discussion}

For early planning of rational rehabilitation treatment to hemiplegic patients after stroke including thalamic

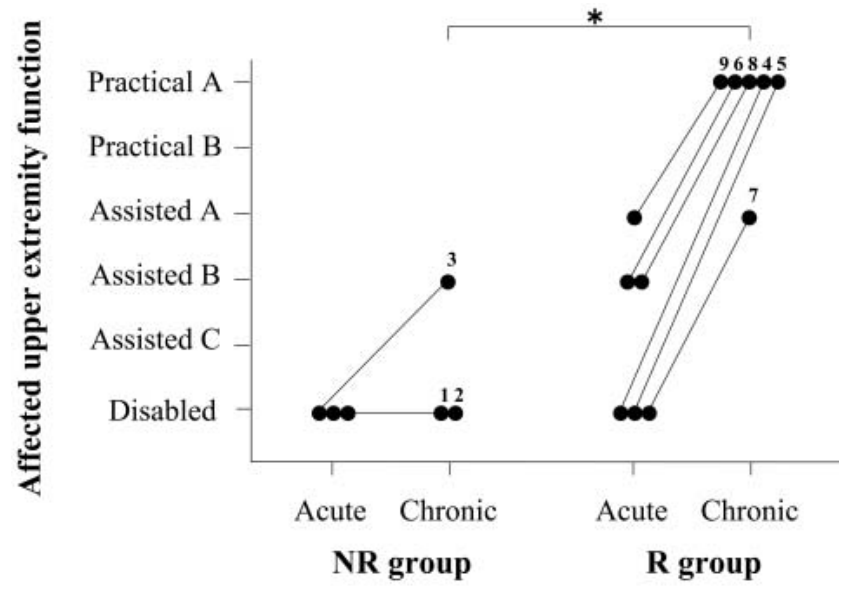

Fig. 5. Relationship between the findings of the median nerve SEFs at the acute stage and the severity of the affected upper extremity function at both the acute and chronic stages. Each number in Fig.5 represents Patient No. Practical A, practical hand A; Practical B, practical hand $\mathrm{B}$, Assisted A, assisted hand A; Assisted B, assisted hand B; Assisted C, assisted hand C; Disabled, disabled hand; *, $\mathrm{p}<0.05$.

hemorrhage, predicting the affected UE function based on a valid prognostic indicator which can be used as soon as possible after stroke onset is needed. Previous studies predicting the outcome of the affected UE function in hemiplegic patients after stroke have reported that the presence of early shoulder shrug ${ }^{20)}$, synergistic hand movements ${ }^{20)}$ or measurable grip strength ${ }^{21) 22)}$ in the affected UE was associated with the good outcome of the affected UE function. Although the prognostic indicators have the advantage of being easy to use clinically, they can not always be applied to hemiplegic patients as soon as possible after stroke onset because they were established in the period of approximately one month after the onset. On the other hand, findings of head diagnostic imaging (especially destruction of the posterior limb of the internal capsule due to a stroke lesion), which is performed immediately after stroke onset for planning of acute medical treatment, may serve as an early prognostic indicator on the affected UE function. However, they are not necessarily associated with functional outcomes ${ }^{5 / 23)}$, as suggested from the results of the present study (see Table and Figures). These problems show the necessity of a new prognostic indicator for early outcome prediction on the affected UE function.

It should be clear that the severities of the motor palsies of the affected UE and fingers are important determinants for the affected UE function in hemiplegic patients. In addition, the severity of the sensory disturbance of the affected UE is also an important one for the affected UE function because sensory feedback is essential to the fine motor control of a UE ${ }^{624)}$. Therefore, it seems reasonable 
to suppose that a prognostic indicator reflecting the outcomes of both the motor palsies and sensory disturbance is used to predict the outcome of the affected UE function in hemiplegic patients. Somatosensory evoked responses (SERs), such as SEFs and somatosensory evoked potentials (SEPs), are objective neurophysiological indicators on sensory functions, and correlations between findings of SERs and outcomes of sensory disturbances have already been reported in several stroke studies ${ }^{4) 624-26)}$. In patients after intracerebral hemorrhage occurring near the posterior limb of the internal capsule such as thalamic hemorrhage, on the other hand, a hemorrhagic lesion and/or perihematomal edema will damage both the corticospinal and somatosensory tracts because they pass the posterior limb of the internal capsule, approaching closely. Therefore, a SER examination would provide useful information on the function of the corticospinal tract as well as that of the somatosensory tract, and permit the outcome prediction on the affected UE function in hemiplegic patients after thalamic hemorrhage ${ }^{9)}$.

The results of the present study indicate that the hemiplegic patients after thalamic hemorrhage showing any median nerve SEF components that originate from the somatosensory cortex in the affected hemisphere and occur between about $20 \mathrm{~ms}$ and $100 \mathrm{~ms}$ post-stimulus at the acute stage (the R group) demonstrate significantly lower severity of the affected UE function at the chronic stage compared with those not showing them (the NR group). Specifically, the affected UE function of almost all the patients in the $\mathrm{R}$ group recovered to "practical hand A", which is the best affected UE function defined by UEAT, at the chronic stage. In contrast, the affected UE function of all the patients in the NR group remained "disabled hand" or recovered only to "assisted hand B" at the chronic stage. Furthermore, the severities of both the motor palsies and sensory disturbance of the affected UE in the R group were also significantly lower than those in the NR group at the chronic stage. These results suggest that the findings of the median nerve SEFs at the acute stage would contribute to the early outcome prediction on the affected UE function as well as the motor palsies and sensory disturbance at the chronic stage in hemiplegic patients after thalamic hemorrhage.

In previous studies using median nerve SEPs on prediction of functional outcomes in hemiplegic patients after thalamic hemorrhage, Maruishi and co-workers ${ }^{6}$ have reported the result conflicting with ours: findings of N20, which is the initial component of median nerve SEPs and corresponds to $\mathrm{N} 20 \mathrm{~m}^{7)}$, at an acute stage were not associated with the outcome of the affected UE function at a chronic stage. However, they did not investigate the relationship between findings of subsequent components after N20 at an acute stage and the outcome of the affected UE function at a chronic stage. Although Misra et al. ${ }^{4}$ has discussed the relationship between findings of median nerve SEPs and functional outcomes, which did not include the outcome of the affected UE function, neither did they perform a detailed investigation of the subsequent components. We suppose two major reasons why the subsequent components were not used in the previous studies. The first is the difficulty of systematic investigation about the subsequent components due to their great interindividual variability ${ }^{1112)}$. The second is the controversy about the source locations of the subsequent components ${ }^{11) 12227)}$. However, patients after thalamic hemorrhage would show only the subsequent components in a SER examination ${ }^{28}$ (This phenomenon was observed in the present study). Furthermore, we would misjudge the prognostic value of SERs if using only initial components of SERs, such as N20 and N20m, because the prognostic value of the subsequent components on functional outcomes has already been reported in recent studies ${ }^{925292}$. Therefore, the subsequent components should be also utilized for prediction of functional outcomes using SERs. In case of this, if the sources of the subsequent components are not located on the somatosensory cortex, the use of the subsequent components as a prognostic indicator should be avoided because their validity as SERs would not be proven from the standpoint of the functional localization in cerebral cortices. We believe that MEG would permit one to show the prognostic value of the subsequent components because it enables their source estimation with high reliability.

One weakness of this study is its small sample size. Additionally, the reliability on the relationship between the findings of the median nerve SEFs at the acute stage and the prognosis of the motor palsies, sensory disturbance and affected UE function at the chronic stage may be reduced by the inconsistency of their severities at the acute stage, although their baseline assessments were performed in this study. Subsequent studies with a larger number of patients, whose severities of the motor palsies, sensory disturbance and affected UE function at the acute stage is strictly controlled, should confirm the validity and reliability of our results. Furthermore, to elucidate the prognostic value of SEFs in other stroke types except thalamic hemorrhage will be also important for the widely application of SEFs to stroke rehabilitation.

\section{References}

1) Omae $T$, Ueda $K$, et al:: Toole TL (ed) Handbook of Clinical Neurology 10. Amsterdam, Elsevier, 1989, pp 287-331.

2) Chui C, Howng SL: Thalamic hemorrhage: a computed tomographic-clinical study in prognostic prediction. Gaoxiong Yi Xue Ke Xue Za Zhi 5: 231-240, 1989.

3) Mori $S$, Sadoshima $S$, et al.: Impact of thalamic hematoma on six-month mortality and motor and cognitive functional outcome. Stroke 26: 620-626, 1995.

4) Misra UK, Kalita J: Evoked potential studies in thalamic hemorrhage. Clin Neurol Neurosurg 98: 291-298, 1996. 
5) Matsuzaki T, Shimazaki M, et al:: Prediction of motor function recovery with coronal MRI in thalamic hemorrhage: Pyramidal line. Brain Hemorrhage 97: 21-27, 1999.

6) Maruishi M, Shima $T$, et al.: Effect of somatosensory disturbance to outcomes of motor function in thalamic hemorrhage: evaluation with short-latency somatosensory evoked potential (SSEP). No To Shinkei 50: 631-635, 1998 (in Japanese).

7) Nakasato N, Yoshimoto T: Somatosensory, auditory, and visual evoked magnetic fields in patients with brain diseases. J Clin Neurophysiol 17: 201-211, 2000.

8) Nakasato $\mathrm{N}$, Itoh $\mathrm{H}$, et al:: Movement-related magnetic fields to tongue protrusion. NeuroImage 14: 924-935, 2001.

9) Yoshida $\mathrm{H}$, Kondo $\mathrm{T}$, et al.: Prediction on early motor recovery in patients with thalamic hemorrhage by using somatosensory evoked magnetic fields. Rigakuryohogaku 31: 1-8, 2004 (in Japanese).

10) Kakigi R: Somatosensory evoked magnetic fields following median nerve stimulation. Neurosci Res 20: 165-174, 1994.

11) Srisa-an P, Lei L, et al.: Somatosensory evoked potentials: noninvasive source analysis. J Clin Neurophysiol 13: 156$163,1996$.

12) Kawamura $T$, Nakasato $N$, et al.: Neuromagnetic evidence of pre- and post-central cortical sources of somatosensory evoked responses. Electroencephalogr Clin Neurophysiol 100: 44-50, 1996.

13) Grynszpan F, Geselowitz DB: Model studies of the magnetocardiogram. Biophys J 13: 911-925, 1973.

14) Hamalainen $M$, Hari $R$, et al:: Magnetoencephalography: theory, instrumentation, and applications to noninvasive studies of the working human brain. Rev Mod Phys 65: 413497, 1993.

15) Wade DT, Wood VA, et al:: Recovery after stroke-The first 3 months. J Neurol Neurosurg Psychiatry 48: 7-13, 1985.

16) Nakayama H, Jorgensen HS, et al:: Recovery of upper extremity function in stroke patients: The Copenhagen stroke study. Arch Phys Med Rehabil 75: 394-398, 1994.

17) Brunnstrom S: Movement Therapy in Hemiplegia; A Neurophysiological Approach. New York, Harpar \& Row,
1970.

18) Hirayama K, Fukutake T, et al.: 'Thumb localizing test' for detecting a lesion in the posterior column-medial lemniscal system. J Neurol Sci 167: 45-49, 1999.

19) Ueda $S$, Hasegawa $T$, et al:: Standardization of the finger function test and upper extremity ability test for hemiplegia. Jpn J Rehabil Med 22: 143-160, 1985 (in Japanese).

20) Katrak P, Bowring G, et al.: Predicting upper limb recovery after stroke: the place of early shoulder and hand movement. Arch Phys Med Rehabil 79: 758-761, 1998.

21) Heller A, Wade DT, et al:: Arm function after stroke: measurement and recovery over the first three months. J Neurol Neurosurg Psychiatry 50: 714-719, 1987.

22) Sunderland A, Tinson D, et al.: Arm function after stroke: An evaluation of grip strength as a measure of recovery and a prognostic indicator. J Neurol Neurosurg Psychiatry 50: 1267-1272, 1989.

23) Feys $\mathrm{H}$, Hetebrij J, et al.: Predicting arm recovery following stroke: value of site of lesion. Acta Neurol Scand 102: 371377, 2000.

24) Kusoffsky A, Wadell I, et al.: The relationship between sensory impairment and motor recovery on patients with hemiplegia. Scand J Rehab Med 14: 27-32, 1982.

25) Wikstrom $\mathrm{H}$, Rione RO, et al.: Specific changes in somatosensory evoked magnetic fields during recovery from sensorimotor stroke. Ann Neurol 47: 353-360, 2000.

26) Huang M, Davis LE, et al:: MEG response to median nerve stimulation correlates with recovery of sensory and motor function after stroke. Clin Neurophysiol 115: 820-833, 2004.

27) Luders H, Lesser RP, et al.: Cortical somatosensory evoked potentials in response to hand stimulation. J Neurosurg 58: 885-894, 1983.

28) Kudo K, Yamadori A: Somatosensory evoked potentials in patients with thalamic hemorrhage. J Neurol 232: 61-66, 1985.

29) Wikstrom H, Rione RO, et al:: Somatosensory evoked magnetic fields from the primary somatosensory cortex (SI) in acute stroke. Clin Neurophysiol 110: 916-923, 1999. 